\title{
Maternal Tobacco Smoking and Decreased Leukocytes, Including Dendritic Cells, in Neonates
}

\author{
JANA M. PACHLOPNIK SCHMID, CLAUDIA E. KUEHNI, MARIE-PIERRE F. STRIPPOLI, HANNA L. ROIHA, \\ RODOLJUB PAVLOVIC, PHILIPP LATZIN, SABINA GALLATI, RICHARD KRAEMER, CLEMENS DAHINDEN, AND URS FREY, \\ on behalf of the SWISS PEDIATRIC RESPIRATORY RESEARCH GROUP \\ Department of Pediatrics [J.M.P.S., H.L.R., P.L., S.G., R.K., U.F.], University Children's Hospital, and Institute of Immunology \\ [R.P., C.D.], Inselspital, 3010 Berne, Switzerland; Department of Social and Preventive Medicine [C.E.K., M-P.F.S.], University of Berne,
} 3012 Berne, Switzerland

\begin{abstract}
Maternal smoking in pregnancy is associated with respiratory diseases in the offspring, possibly due to prenatal influences on the developing immune system. We investigated whether maternal smoking in pregnancy was associated with cord blood leukocyte numbers, including precursor dendritic cells, adjusting for concomitant factors. In a prospective healthy birth cohort study, total leukocyte counts were reduced in neonates of smoking mothers [10.7 (8.4-13.0), $n=14]$ compared with nonexposed infants [14.7 (13.715.7), $n=74, p=0.002$ ] [geometric mean cells $\times 10^{3} / \mu \mathrm{L}(95 \%$ confidence interval)]. All leukocyte subsets were decreased, most prominently segmented neutrophils [4.3 (2.8-5.7) versus 6.2 (5.5$6.8), p=0.021]$, lymphocytes [3.8 (2.9-4.8) versus 5.0 (4.5-5.6), $p=0.036]$, and myeloid precursor dendritic cells $[12.7$ cells $/ \mu \mathrm{L}$ (9.1-17.8) versus $18.3(15.8-21.2), p=0.055]$. These differences persisted after adjustment for possible confounders. Predictors of myeloid precursor dendritic cell numbers in multivariable models were maternal smoking $(-5.1$ cells $/ \mu \mathrm{L}, p=0.042)$, age $(-0.5$ cells/ $\mu \mathrm{L} / \mathrm{y}, p=0.035)$, and, marginally, asthma $(+8.1$ cells $/ \mu \mathrm{L}, p=$ 0.075). The decrease of all leukocytes in neonates of smoking mothers could be clinically significant and suggests a decreased cell production, increased peripheral recruitment, or retention in bone marrow. Given the importance of dendritic cells in early immune responses, their decrease might reflect an impact of maternal smoking on the developing fetal immune system. (Pediatr Res 61: 462-466, 2007)
\end{abstract}

$\mathrm{E}$ nvironmental tobacco smoke exposure is associated with an increased risk of infections, impaired lung development, and respiratory morbidity and mortality in children (1). Specifically, smoking during pregnancy has been shown to act as an independent risk factor for wheezing disorders when compared with postnatal exposure to environmental tobacco smoke (2). Whereas inhaled tobacco smoke is known to have an effect on leukocyte numbers in the blood and to induce inflammatory responses in the airways of human adults, the

Received September 5, 2006; accepted November 29, 2006.

Correspondence: Urs Frey, M.D., Ph.D., Pediatric Respiratory Medicine, Department of Pediatrics, University Hospital Inselspital, 3010 Berne, Switzerland; e-mail: urs.frey@insel.ch

This work was supported by the Swiss National Foundation grant no. 3233-069348 and 3200-069349 to C.E.K., grant no. 32-68025.02 to U.F., the Swiss Foundation for Grants in Medicine and Biology grant no. 1211/PASMA-110658/1, a grant from the University Children's Hospital Foundation, and a grant from the Fondazione Ettore e Valeria Rossi to J.M.P.S.

DOI: $10.1203 /$ pdr.0b013e3180332d02 effects of maternal smoking on blood counts and early immune responses of the offspring are poorly understood.

Studies showing a decrease in leukocyte counts, especially neutrophilic granulocytes, in cord blood of neonates of smoking mothers have lead to the conclusion that this decrease might contribute to the susceptibility to infections and therefore increased respiratory morbidity in these children $(3,4)$. However, these studies used only univariate statistical methods and were therefore not able to determine whether the decreased leukocyte counts were independently associated with maternal smoking, or whether they could be explained by other factors (e.g. maternal age or perinatal stress) that might be associated with both, maternal smoking and neonatal cell counts.

The decrease in the number of neutrophilic granulocytes might contribute to an increased susceptibility to bacterial infections, whereas it is unlikely to be the major cause for the increased susceptibility to viral infections that are associated with symptoms of wheeze. Not neutrophils but other leukocytes such as lymphocytes and antigen-presenting dendritic cells play a more important role in the defense against most viral infections. Dysregulation of these cells may furthermore contribute to the development of allergy and asthma $(5,6)$. Nicotine, one of the main constituents of cigarette smoke, has a suppressive effect on myeloid dendritic cells (7-9). As a consequence, these cells may be particularly affected in children of smoking mothers.

The aim of this study was to investigate whether maternal smoking in pregnancy was associated with a decrease of lymphocytes and dendritic precursor cells in the cord blood of the neonates and whether these differences in leukocyte subtypes remained after adjustment for other determinants of neonatal leukocyte counts, such as maternal age, infections, atopic diseases, and perinatal stress.

\section{MATERIALS AND METHODS}

Subjects and protocol. In a prospective birth cohort study, 97 Caucasian infants were prenatally recruited from seven maternity hospitals in the region of Berne, Switzerland, between August 1999 and May 2005. Exclusion criteria for the study were as follows: ethnicity other than Caucasian, signif-

Abbreviations: CD, cluster of differentiation; pDC, precursor dendritic cells; Th, T helper lymphocyte 
icant preterm delivery ( $<36 \mathrm{wk}$ ), major birth defects, respiratory distress with need of supplemental oxygen longer than $30 \mathrm{~min}$ after birth, or other significant perinatal diseases, e.g. severe neonatal or maternal infections. The infants were part of a larger sample reported previously $(10,11)$. A few days after birth, an extensive questionnaire was completed with the mothers by standardized interviews. Included were questions related to maternal and paternal tobacco smoking, maternal infections (of airways, gastrointestinal tract, urinary tract, vagina, or others) and medications in each trimester. Information about perinatal circumstances was obtained from the medical record. History of parental atopic disease (self-reported, doctor-diagnosed asthma, hay fever, or eczema) was verified by standardized interviews by a pediatric pulmonologist (U.F.) (Table 1). Informed consent was obtained from the children's parents. The study protocol was approved by the Departmental Ethics Committee of the University Children's Hospital and by the Governmental Ethics Committee of the State of Berne, Switzerland.

Measurements. Cord blood was taken by venipuncture from the umbilical cord attached to the placenta, immediately following separation from the infant and collected in sterile EDTA tubes. Blood analyses were performed within $12 \mathrm{~h}$ after birth. Complete blood counts (except for pDC) were determined by the hematological laboratory of the University Children's Hospital Berne using a Cell-Dyn 3500R (Abbott, Baar, Switzerland). Dendritic precursor cells were flow cytometrically measured using a FACScan (BD Biosciences, Franklin Lakes, NJ) and were identified as follows: 1) gating for mononuclear cells in forward and orthogonal light scattering signals; 2) selection of cells within the gated population by four color-flow cytometry: 2a) either CD 11c (myeloid) or CD123 (lymphoid) highly positive; 2b) HLA-DR positive; 2c) CD3, CD14, CD16, CD19, CD20, and CD56 negative.

The following monoclonal mouse anti-human antibodies were used: antiCD11c allocyanin (APC), anti-CD123 phycoerythrin (PE), lineage cocktail (lin 1) FITC (anti-CD3, CD14, CD16, CD19, CD20, and CD56), and antiHLA-DR peridinin chlorophyll protein (PerCP). Mouse anti-human $\operatorname{IgG}_{2 \mathrm{a}}$ APC, PerCP, FITC, and mouse anti-human $\mathrm{IgG}_{1}$ PE were used as isotype controls. The antibodies were purchased from BD Biosciences. Contamination by maternal blood was detected by measurement of IgA. Children with measurable IgA in cord blood sample were excluded from the study. Total leukocyte counts were corrected for nucleated red cells.

Cotinine levels were measured in the first urine of the neonates (or in the second if the first was contaminated by meconium) by rapid gas-liquid chromatography (12). Cotinine levels depended on the timing of the last cigarette in relation to the collection of the urine sample and were not strongly associated with the mean cigarette consumption of the mother during pregnancy, because several mothers had stopped or greatly reduced smoking in the days preceding delivery and because the time between birth and collection of the first urine varied. Further validation by home visits showed that reported smoking reflected long-term exposure better than the postnatal urinary cotinine level. We therefore based our analyses on reported smoking frequency, but used cotinine levels to detect underreporting of smoking (e.g. children with a negative history and high urinary cotinine). This led to the exclusion of one child from the analyses.

Data analysis. Cord blood cell counts from 88 out of 97 infants could be included in the analyses; seven blood samples were coagulated, and one child was excluded because the mother's smoking history (nonsmoker) was contradicted by a high urine cotinine level $(93 \mathrm{ng} / \mathrm{mL})$. An otherwise healthy infant of a smoking mother was excluded due to marked leucopenia of unknown origin $\left(2.0 \times 10^{3}\right.$ cells $\left./ \mu \mathrm{L}\right)$ [age-matched reference values (10th90th percentiles): $7.20-18.00 \times 10^{3}$ cells $/ \mu \mathrm{L}$ (13)].

Analyses were performed with STATA, version 8.2 for Windows (STATA Corporation, Austin, TX). Cell counts were inspected for normality and, if necessary, transformed before analysis. Associations between maternal smoking and cell counts were first analyzed by means of $t$ tests and univariate linear regression, with results expressed as means (95\% confidence intervals). To assess whether these effects could be explained by confounding, the regression models were then adjusted for a number of factors selected a priori as potential determinants of neonatal cell counts (Table 2) even though most of them did not independently affect cell counts and were only weakly associated with maternal smoking. Finally, in view of the small sample size, we restricted the model for $\mathrm{pDC}$ to include only covariates that were found to significantly predict cell counts $(p<0.1)$ in the multivariable model. Estimated effects of these predictors and of maternal smoking were reported in terms of deviation from mean cell counts in children with baseline characteristics. For all regression models, continuous variables (gestational age, birth weight, maternal age at birth) were centred and categorical variables were entered as indicator variables.

\section{RESULTS}

Fourteen of 88 mothers had smoked during pregnancy. Prevalence of other potential determinants of cord blood leukocytes in smokers and nonsmokers are summarized in Table 1. Other factors that were looked at but did not show any association with leukocyte numbers or smoking history were Streptococcus type B on vaginal smear $(n=2)$, maternal use of inhaled steroids $(n=2)$, and coffee consumption during pregnancy $(n=55)$. Apart from smoking, maternal histories for drug abuse were negative. Blood pressure values of all mothers were within the normal range.

Whereas cord blood $\mathrm{Hb}$ and thrombocytes were not associated with maternal smoking (Table 2, unadjusted), total leukocyte counts were significantly lower $\left(10.7 \times 10^{3} / \mu \mathrm{L}\right)$ in cord blood of children of smoking mothers compared with those of nonsmokers $\left(14.7 \times 10^{3} / \mu \mathrm{L}\right)(p=0.002)$. Within the leukocyte range, the most prominent decrease was observed in segmented neutrophilic granulocytes, monocytes, myeloid pDCs, and lymphocytes (Table 2). Mean lymphocyte counts in cord blood of children of smoking mothers were $3.8 \times 10^{3}$ cells $/ \mu \mathrm{L}$, in those of nonsmokers $5.0 \times 10^{3}$ cells $/ \mu \mathrm{L}$ [agematched reference values (10th-90th percentiles): 3.40-7.60 $\times$ $10^{3}$ cells $/ \mu \mathrm{L}$ (13)]. These differences remained essentially unchanged after adjustment for all potential confounders (Table 2 , adjusted values). When we included the child with

Table 1. Characteristics of the study population, by maternal smoking

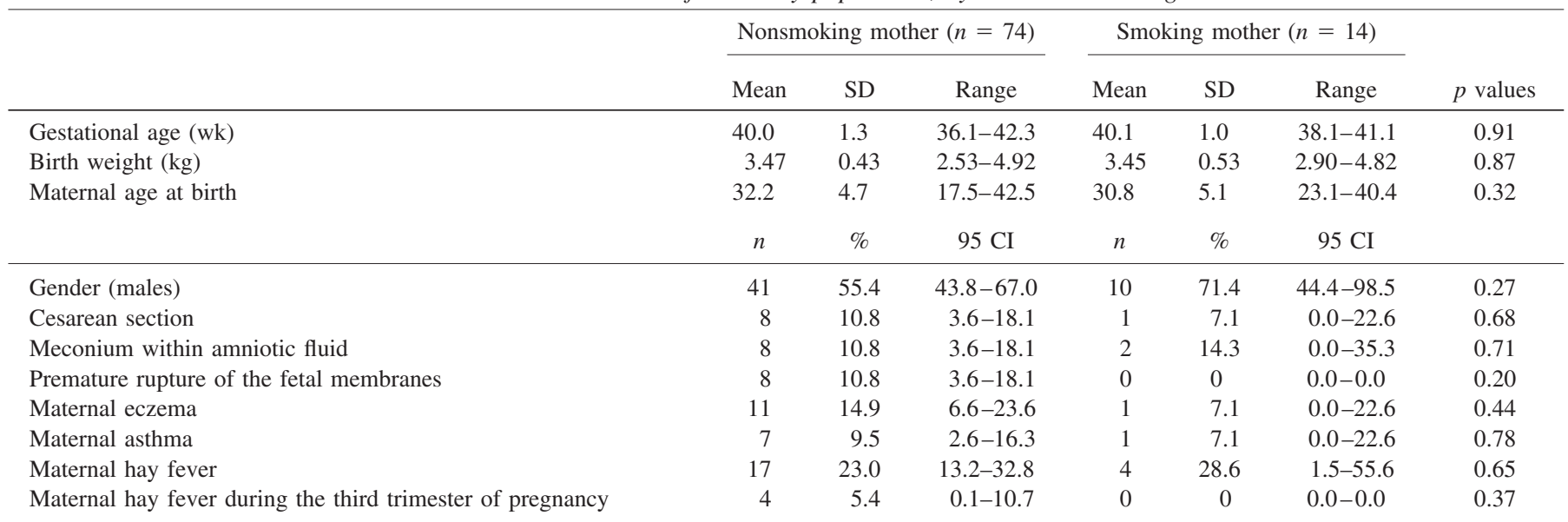


Table 2. Cell counts in cord blood of neonates of nonsmoking and smoking mothers, unadjusted and adjusted for potential confounding factors

\begin{tabular}{|c|c|c|c|c|c|c|c|c|c|c|c|c|}
\hline & \multicolumn{7}{|c|}{ Cell counts, unadjusted } & \multicolumn{5}{|c|}{ Cell counts, adjusted* } \\
\hline Total leukocytes $\ddagger$ & 74 & 14.7 & $(13.7-15.7)$ & 14 & 10.7 & $(8.4-13.0)$ & 0.002 & 15.0 & $(13.4-16.7)$ & 11.2 & $(8.6-13.9)$ & 0.002 \\
\hline Neutrophilic granulocytes & & & & & & & & & & & & \\
\hline Banded neutrophilsł & 64 & 0.8 & $(0.6-1.0)$ & 13 & 0.5 & $(0.3-0.8)$ & 0.075 & 0.9 & $(0.6-1.2)$ & 0.6 & $(0.3-1.0)$ & 0.136 \\
\hline Basophilic granulocytes§ & 69 & 57.1 & $(49.7-65.6)$ & 13 & 41.1 & $(29.9-56.7)$ & 0.066 & 52.2 & $(41.1-66.2)$ & 36.9 & $(24.8-54.8)$ & 0.063 \\
\hline Monocytes & 64 & 1.3 & $(1.1-1.5)$ & 13 & 1.0 & $(0.7-1.3)$ & 0.104 & 1.3 & $(1.0-1.6)$ & 0.9 & $(0.6-1.3)$ & 0.049 \\
\hline Lymphocytes $\ddagger$ & 64 & 5.0 & $(4.5-5.6)$ & 13 & 3.8 & $(2.9-4.8)$ & 0.036 & 4.9 & $(4.1-5.8)$ & 3.7 & $(2.6-5.0)$ & 0.047 \\
\hline CD11c + myeloid pDCs§ & 69 & 18.3 & $(15.8-21.2)$ & 13 & 12.7 & $(9.1-17.8)$ & 0.055 & 15.9 & $(12.4-20.4)$ & 11.0 & $(7.2-16.6)$ & 0.055 \\
\hline CD123 + lymphoid pDCs§ & 69 & 12.8 & $(11.0-14.9)$ & 13 & 9.5 & $(6.7-13.5)$ & 0.125 & 11.0 & $(8.5-14.3)$ & 7.9 & $(5.1-12.1)$ & 0.093 \\
\hline Thrombocytes $\ddagger$ & 57 & 230 & $(279-315)$ & 9 & 262 & $(216-308)$ & 0.160 & 300 & $(269-332)$ & 268 & $(211-325)$ & 0.233 \\
\hline
\end{tabular}

* Adjusted for gender of child, gestational age, birth weight, cesarean section, meconium within the amniotic fluid, premature rupture of fetal membranes, age of the mother at birth, maternal asthma and hay fever ever in life, and maternal hay fever during the third trimester of pregnancy.

$\dagger$ Total $n$ differed slightly between different cell types because some analyses could not be performed in children born during weekends.

$\doteqdot$ Cells $\times 10^{3} / \mu \mathrm{L}$

$\S$ Cells $/ \mu \mathrm{L}$.

leucopenia in the analyses, the effect of smoking was even larger (data not shown).

Apart from maternal smoking, other independent predictors $(p<0.1)$ of myeloid pDC numbers in a more restricted multivariable model were maternal age $(-0.5$ cells $/ \mu \mathrm{L}$ per year of maternal age, $p=0.035)$ and, marginally, maternal asthma $(+8.1 \mathrm{cells} / \mu \mathrm{L}, p=0.075)$ (Table 3$)$. Predictors of lymphoid $\mathrm{pDC}$ numbers included maternal hay fever during the third trimester of pregnancy $(+8.2$ cells $/ \mu \mathrm{L}, p=0.074)$ and male gender $(+3.0$ cells $/ \mu \mathrm{L}, p=0.077)$.

Paternal smoking history per se was not associated with cell counts in cord blood, but it is noteworthy that the fathers mainly smoked outside the house if the mother was a nonsmoker, whereas they smoked inside when the mother was a smoker. Thus, smoking pregnant women additionally had an increased exposure to environmental tobacco smoke.

\section{DISCUSSION}

In this birth cohort study of unselected infants, we found a decrease in all leukocyte subtypes in the cord blood of neonates of mothers who smoked during pregnancy compared with infants of nonsmoking mothers. This decrease was most prominent in segmented neutrophilic granulocytes, mono- cytes, lymphocytes, and myeloid pDCs and remained essentially unchanged even after adjustment for a large number of potential confounders. The discrepancies found were large enough to have potential clinical significance and therefore call for further investigation.

The observed decrease in all subtypes of the leukocyte lineage suggests either a reduced production of these cells, diminished release from bone marrow, or increased migration into other organs, such as the lung. Others have shown that reticulocyte counts in cord blood of children of smoking mothers were lower than in unexposed newborns, even though $\mathrm{Hb}$ did not differ between the two groups (4). This would be in accordance with a reduced bone marrow activity in response to maternal tobacco smoke exposure. Platelet counts in these children did not differ by maternal smoking status (4). In our study, the decrease of segmented (mature) neutrophils in children of smoking mothers was larger than the one of banded neutrophils. This could be explained by an increased recruitment of mature neutrophils to some tissues. In adults, acute and chronic smoking leads in fact to an increased number of neutrophils in bronchoalveolar lavage fluids (14). An accumulation of neutrophils in the airways could furthermore be explained by the inhibition of neutrophilic chemo-

Table 3. Factors associated with myeloid and lymphoid pDC counts in cord blood (multiple linear regression, adjusted for all variables listed; $n=82$ )

\begin{tabular}{|c|c|c|c|c|}
\hline & \multicolumn{2}{|c|}{ CD11c + myeloid pDCs } & \multicolumn{2}{|c|}{ CD123+ lymphoid pDCs } \\
\hline Maternal smoking & -5.1 & 0.042 & -2.9 & 0.089 \\
\hline Maternal asthma & 8.1 & 0.075 & 4.0 & 0.165 \\
\hline Hay fever during third trimester of pregnancy & 9.6 & 0.136 & 8.2 & 0.074 \\
\hline Male gender & 1.7 & 0.460 & 3.0 & 0.077 \\
\hline
\end{tabular}

According to the model, children with baseline characteristics (female, no maternal smoking, average age of mother at birth: $31 \mathrm{y}$, no maternal asthma, no maternal hay fever during the third trimester of pregnancy) have counts of 16.3 cells $/ \mu \mathrm{L}$ and 10.6 cells $/ \mu \mathrm{L}$, for myeloid and lymphoid pDCs, respectively. 
taxis by cigarette smoke components, thus "trapping" of the neutrophils within the airways (15). Obviously, administration routes of the substances from cigarette smoke differ among adult smokers and fetuses exposed to maternal smoking and the mechanism by which maternal smoking affects fetal neutrophils remains speculative. However, mononuclear cells from smokers have an increased production of certain cytokines and an increased proliferative response to mitogens (16). Dendritic cells consistently repopulate normal tissues from the bloodstream and are recruited in elevated numbers to the sites of inflammation (17). One could hypothesize that maternal smoking induces neonatal inflammatory cellular reactions that lead to an increased recruitment of dendritic cells and other leukocytes into periphery and thus a reduction of their number in the cord blood. Once recruited, these cells may, due to the effect of the constituents of cigarette smoke (7-9) show a dysbalanced immune reaction.

Environmental factors influence the immature immune system in utero and could contribute to a failure of development of immune functions $(18,19)$. It is noteworthy that pDCs, particularly myeloid pDCs, and monocytes, the putative precursors of myeloid pDCs, were decreased in newborns of mothers who smoked during pregnancy. In view of the importance of myeloid dendritic cells in the activation of adaptive immune responses, our study suggests that maternal smoking might further accentuate the physiologic immaturity and Th2 bias of the immune system of neonates. Lymphocyte function has been shown to be altered in newborns of smoking mothers (20). Furthermore, nicotine is known to affect the myeloid dendritic cell function, which may lead to a suppression of Th1 polarization (7). Thus, the decreased pDC number could reflect an important alteration in the developing immune system. This may be relevant already during pregnancy, since many studies suggest that initial priming of the $T$ cell system toward atopic disease takes place in the fetal period (5). Because all of the mothers that were included in this study smoked during all three trimesters of the pregnancy, we cannot draw conclusions about the most vulnerable period for prenatal nicotine exposure. Much larger birth cohorts would be needed to tackle this question.

Because dendritic cells have a central role in initiating immune responses and nicotine is known to have a suppressive effect on myeloid dendritic cells, this may be relevant for the pathophysiology of diseases potentially induced by fetal immune dysregulations such as bronchial asthma. Maternal smoking has been shown to be associated with increased cord blood mononuclear cell proliferative response to stimulation with certain allergens $(20,21)$. We have not measured proliferative response to allergen stimulation in our cohort, and the link between our findings and these publications remains hypothetical. The decrease in the number of lymphocytes that we have found in the cord blood of neonates of smoking mothers could further reflect an altered adaptive immune response in these children.

Strengths and limitations. This is the first study in humans showing that maternal smoking in pregnancy is associated with decreased pDCs in the offspring, and that these findings remain unchanged after adjustment for a large number of concomitant factors. A limitation of the study, due to its population-based sampling design, is the relatively low number of infants exposed to maternal smoking during pregnancy (16\% of the study population), which limits statistical power for the analysis. Therefore, the results, especially for pDC, need to be confirmed by other studies with independent and preferentially larger groups of children. Also, it will be important to monitor the long-term clinical outcome of these infants, to assess if neonates with decreased cell counts have in fact an increased risk to develop respiratory symptoms in infancy and early childhood. We could not confirm in this study the reported increased risk of low birth weight and preterm birth associated with maternal smoking (22) because of the limited number of cases and, in consequence, insufficient statistical power to assess slight differences $(-200 \mathrm{~g})$ in mean weight; the fact that most of the mothers smoked little ( $<10$ cigarette/d); and the fact that we had excluded preterm babies from participation in the study.

Summary and implications. We have shown that maternal smoking in pregnancy was associated with reduced counts of all cell subpopulations deriving from the myeloid and lymphoid lineage in neonates, especially segmented neutrophilic granulocytes, monocytes, myeloid pDCs, and lymphocytes. These findings, if confirmed by other groups, suggest a common pathway, with perhaps different susceptibility of these cell subtypes to tobacco smoke components. These findings represent a further step toward identifying potential in utero mechanisms linking maternal tobacco smoking during pregnancy to infants' susceptibility to infections and respiratory morbidity. Because dendritic cells particularly might have a central role in the immune development of the offspring and since nicotine is known to have a suppressive effect on myeloid dendritic cells, this may be relevant for the pathophysiology of diseases potentially induced by fetal immune dysregulations such as bronchial asthma. Future studies, investigating the effect of maternal tobacco smoke exposure on the redirection of the neonatal, Th2 skewed, immune response toward the Th1 cytokine phenotype, may be of particular interest, providing more insight into the mechanisms leading to respiratory morbidity in these children.

Acknowledgments. The authors thank the study children and their families for their cooperation and also the staff of the hematological laboratory of the University Children's Hospital Berne for their appreciated collaboration. We also thank Christine Becher, Helen Gehr, Monika Graf, and Margrith Otth for their excellent technical assistance.

\section{REFERENCES}

1. Cook DG, Strachan DP, Carey IM 1998 Health effects of passive smoking. Thorax 53:884-893

2. Gilliland FD, Berhane K, McConnell R, Gauderman WJ, Vora H, Rappaport EB, Avol E, Peters JM 2000 Maternal smoking during pregnancy, environmental tobacco smoke exposure and childhood lung function. Thorax 55:271-276

3. Harrison KL 1979 The effect of maternal smoking on neonatal leukocytes. Aust N Z J Obstet Gynaecol 19:166-168

4. Mercelina-Roumans PE, Breukers RB, Ubachs JM, van Wersch JW 1996 Hematological variables in cord blood of neonates of smoking and nonsmoking mothers. J Clin Epidemiol 49:449-454 
5. Holt PG, Upham JW, Sly PD 2005 Contemporaneous maturation of immunologic and respiratory functions during early childhood: implications for development of asthma prevention strategies. J Allergy Clin Immunol 116:16-24

6. Kuipers H, Lambrecht BN 2004 The interplay of dendritic cells, Th2 cells and regulatory $\mathrm{T}$ cells in asthma. Curr Opin Immunol 16:702-708

7. Nouri-Shirazi M, Guinet E 2003 Evidence for the immunosuppressive role of nicotine on human dendritic cell functions. Immunology 109:365-373

8. Vassallo R, Chen L 2004 Selective suppression of dendritic cell functions by cigarette smoke extract. Chest 125:107S

9. Robbins CS, Dawe DE, Goncharova SI, Pouladi MA, Drannik AG, Swirski FK, Cox G, Stampfli MR 2004 Cigarette smoke decreases pulmonary dendritic cells and impacts antiviral immune responsiveness. Am J Respir Cell Mol Biol 30:202-211

10. Frey U, Kuehni C, Roiha H, Cernelc M, Reinmann B, Wildhaber JH, Hall GL 2004 Maternal atopic disease modifies effects of prenatal risk factors on exhaled nitric oxide in infants. Am J Respir Crit Care Med 170:260-265

11. Hall GL, Reinmann B, Wildhaber JH, Frey U 2002 Tidal exhaled nitric oxide in healthy, unsedated newborn infants with prenatal tobacco exposure. J Appl Physiol 92:59-66

12. Feyerabend C, Russell MA 1990 A rapid gas-liquid chromatographic method for the determination of cotinine and nicotine in biological fluids. J Pharm Pharmacol 42:450-452

13. Shearer WT, Rosenblatt HM, Gelman RS, Ovomopito R, Plaeger S, Stiehm ER, Wara DW, Douglas SD, Luzuriaga K, Mc Farland EJ, Yogev R, Rathore MH, Levy W, Graham BL, Spector SA 2003 Lymphocyte subsets in healthy children from birth through 18 years of age: the Pediatric AIDS Clinical Trials Group P1009 study. J Allergy Clin Immunol 112:973-980
14. Morrison D, Rahman I, Lannan S, MacNee W 1999 Epithelial permeability, inflammation, and oxidant stress in the air spaces of smokers. Am J Respir Crit Care Med 159:473-479

15. Bridges RB, Hsieh L 1986 Effects of cigarette smoke fractions on the chemotaxis of polymorphonuclear leukocytes. J Leukoc Biol 40:73-85

16. Zeidel A, Beilin B, Yarendi I, Mayburd E, Smirnov G, Bessler H 2002 Immune responses in asymptomatic smokers. Acta Anaesthesiol Scand 46:959-964

17. Cavanagh LL, Von Andrian UH 2002 Travellers in many guises: the origin and destinations of dendritic cells. Immunol Cell Biol 80:448-462

18. Koning H, Baert MR, Oranje AP, Savelkoul HF, Neijens HJ 1996 Development of immune functions related to allergic mechanisms in young children. Pediatr Res 40:363-375

19. Noakes PS, Hale J, Thomas R, Lane C, Devadason SG, Prescott SL 2006 Maternal smoking is associated with impaired neonatal Toll-like receptor (TLR) mediated immune responses. Eur Respir J 28:721-729

20. Devereux G, Barker RN, Seaton A 2002 Antenatal determinants of neonatal immune responses to allergens. Clin Exp Allergy 32:43-50

21. Willwerth BM, Schaub B, Tantisira KG, Gold DR, Palmer LJ, Litonjua AA, Perkins DL, Schroeter C, Gibbons FK, Gillman MW, Weiss ST, Finn PW 2006 Prenatal, perinatal, and heritable influences on cord blood immune responses. Ann Allergy Asthma Immunol 96:445-453

22. Lumley J, Oliver SS, Chamberlain C, Oakley L 2004 Interventions for promoting smoking cessation during pregnancy. Cochrane Database Syst Rev 18:CD001055. 